\title{
EFECTOS DE LOS INCENDIOS FORESTALES EN LA VEGETACIÓN Y EL SUELO EN LA CUENCA MEDITERRÁNEA: REVISIÓN BIBLIOGRÁFICA
}

\author{
Merche B. Bodí ${ }^{1}$, , 3 , Artemi Cerdà ${ }^{1}$, Jorge Mataix-Solera², Stefan H. Doerr ${ }^{3}$ \\ 'Departamento de Geografía, Universidad de Valencia \\ ${ }^{2}$ Departamento de Agroquímica y Medio Ambiente, Universidad Miguel Hernández \\ ${ }^{3}$ School of the Environment and Society, Swansea University
}

\section{RESUMEN}

Los ecosistemas mediterráneos son susceptibles a los incendios forestales y han evolucionado con la recurrencia de este factor ecológico natural desde el Plioceno. Con el control del fuego por el ser humano se incrementó la frecuencia de los incendios para gestionar el entorno. Sin embargo, la actual despoblación y abandono de la montaña mediterránea europea ha provocado un aumento de la cubierta vegetal y un incremento de incendios forestales sin control y de alta intensidad. Esta revisión bibliográfica muestra que después de un incendio forestal en el Mediterráneo, el recubrimiento vegetal es relativamente rápido y que los efectos del fuego en el suelo y las tasas de erosión no suelen ser catastróficos excepto el primer año. No obstante, los efectos pueden ser muy variables según la intensidad y severidad del fuego y por este motivo no siempre es necesaria una actuación post-incendio. Se concluye que se necesita una adecuada gestión del monte en la que no se obvie el fuego como elemento clave de los ecosistemas mediterráneos.

Palabras clave: fuego, vegetación, suelo, ecosistemas mediterráneos.

\section{ABSTRACT}

A review of fire effects on vegetation and soil in the Mediterranean basin. Mediterranean ecosystems are prone to forest fires and its recurrence played an important role in the evolution of these ecosystems. In addition, fires were more frequent after humans started controlling

Fecha de recepción: febrero 2010.

Fecha de aceptación: febrero 2012. 
them with the aim of managing the landscape. On the contrary, the current depopulation and abandonment of the European Mediterranean forests resulted in an increase of the vegetation cover leading to high intensity fires that burn out of control. This bibliographic revision shows that after a forest fire, the vegetation recovery is relatively fast and fire effects on soil and erosion rates are not catastrophic except the first year. However, the effects of fire in the ecosystems are different depending on the intensity and severity of the fire. For this reason, post-fire treatments are not always necessary. As a conclusion, an appropriate forest management that does not obviate the fire as a key element of the Mediterranean ecosystems is needed.

Key words: fire, vegetation, soil, Mediterranean ecosystems.

\section{INTRODUCCIÓN}

El fuego tiene un importante papel en los procesos que gestionan el Sistema Tierra. La abundancia de carbones en los sedimentos demuestra que el fuego ha actuado desde hace 400 millones de años (principios del Devónico) cambiando su frecuencia e intensidad según los niveles de oxígeno atmosférico y el clima. Como componente del sistema terrestre, el fuego ha influido en la composición de los gases de la atmósfera, el clima, la biota, las geoformas, el transporte de materiales y las tasas de sedimentación (Scott, 2000; 2009).

La configuración de ecosistemas como los bosques boreales de coníferas, praderas, sabanas y bosques mediterráneos, es debida principalmente a la acción del fuego además de por motivos climáticos (Bond et al. 2004, Bodí et al. 2008). La recurrencia de incendios propicia un ecosistema distinto del esperado según la situación climática en la que se encuentra. En estas circunstancias de incendios reiterados, las especies con algún mecanismo de resistencia al fuego perduran y desarrollan para su propia supervivencia dispositivos de reproducción y morfologías para resistir e incluso favorecer los incendios con un régimen concreto de recurrencia (Pyne, 2001). El fuego es así un factor ecológico esencial en la distribución de los biomas de la Tierra, funcionando como un gran herbívoro (Bond y Keeley, 2005). A escala humana y regional los efectos de los incendios son heterogéneos y a veces catastróficos. No obstante, incluso dentro una misma zona, según la litología, régimen de precipitación, orientación o usos del suelo, los efectos serán variados y contrastados (Neary et al., 1999; Kutiel, 2006).

Esta importancia y complejidad de los incendios forestales hace que su estudio sea una cuestión relevante en determinadas zonas del mundo. En Valencia, ya hace 20 años se llevó a cabo el seminario «El papel del fuego en los ecosistemas mediterráneos» organizado por Manuel Costa en la Universidad Internacional Menéndez Pelayo. En éste, se avanzaron las investigaciones más actualizadas sobre la cuestión con la presencia de prestigiosos científicos que trabajaban (y siguen trabajando) en el estudio de los incendios forestales en ecosistemas mediterráneos: P. Trabaud, Z. Naveh, A. Pons, P. Zedler, W. Oechel y W. Christensen entre otros. Además del papel de los incendios forestales en el mediterráneo, se trataron otros temas relacionados como los efectos de los incendios en el suelo, vegetación, fauna, gestión pre- y post- incendio e incluso se adelantaron problemas tan actuales como el incremento de $\mathrm{CO}_{2}$ en la atmosfera. 
En este momento, la gestión del monte con relación a los incendios forestales sigue siendo un tema de actualidad en el que todavía queda mucho por aprender. Un ejemplo es la existencia de FUEGORED, una red temática nacional sobre los efectos de los incendios forestales en el suelo (http://grupo.us.es/fuegored), subvencionada por el Ministerio de Ciencia e Innovación y de la que forman parte la mayoría de científicos que trabajan en este tema en la Península Ibérica. En este contexto organizan colaboraciones, reuniones científicas anuales y publicaciones (ver Cerdà y Mataix coord., 2009 y Cerdà y Jordán coord., 2010). El objetivo de la presente revisión intenta resumir la investigación llevada a cabo desde aquel seminario hasta ahora, tras tres reuniones de FUEGORED, sobre los efectos de los incendios forestales en la cuenca mediterránea, centrándose en los efectos en la vegetación, el suelo y los procesos hidrológicos en el este de la Península Ibérica.

\section{LOS INCENDIOS FORESTALES EN LA CUENCA MEDITERRÁNEA}

Los ecosistemas mediterráneos poseen un clima de transición entre el régimen templado oceánico y el tropical seco. Su característica más importante es la coincidencia de la época seca con la cálida. En verano, el Mediterráneo se encuentra bajo la influencia de los anticiclones subtropicales secos que se retiran en invierno hacia latitudes más bajas dejando paso a las borrascas atlánticas de latitudes templadas. Esta peculiaridad propicia que los ecosistemas sean susceptibles a los incendios forestales con una vegetación agostada en verano, que junto a su naturaleza inflamable favorece la aparición y expansión de los incendios (Naveh, 1991; Arianoutsou et al., 1993).

El fuego ha estado presente en el Mediterráneo como fenómeno natural mucho antes de que el hombre existiera, ya fueran provocados por rayos en tormentas eléctricas o debido a erupciones volcánicas (Naveh, 1975). De hecho, los incendios fueron durante el Plioceno uno de los factores que contribuyeron a configurar, junto al clima, la vegetación mediterránea (Bond y Keeley, 2005; Mataix-Solera y Guerrero, 2007). No se sabe con exactitud cuál era el régimen de incendios natural, pero debió caracterizarse por incendios menos frecuentes y áreas afectadas más grandes, ya que el fuego actuaba sobre un paisaje más homogéneo que el actual y sin barreras antrópicas como carreteras, ciudades, pastos o campos de cultivo (Naveh, 1975; Arianoutsou et al., 1993).

Con la aparición de las sociedades de cazadores-recolectores se utiliza el fuego para abrir claros en el bosque y crear zonas más accesibles, favorecer la producción de ciertos alimentos y propiciar mejores zonas de caza (Naveh, 1991). Pero el mayor impacto del uso del fuego se produjo durante el Neolítico. El hombre se convierte en agricultor y ganadero, y necesita amplias zonas para pastos y cultivo que consigue al deforestar mediante el fuego. Estos incendios controlados, denominados rozas, se han seguido practicando hasta los años 60 en España y en la Europa Mediterránea también con otros fines: controlar plagas y malas hierbas, fertilizar con cenizas, mejorar pastos y modificar el bosque (Naveh, 1974; Dupré, 1983; Carcaillet et al., 2002).

Análisis palinológicos y arqueológicos demuestran la utilización del fuego desde hace al menos 7000 años en el territorio valenciano. Antes del uso del fuego como herramienta, predominaba el bosque mediterráneo de Quercus ilex y Quercus faginea, donde el Pinus $s p$. predominaba en las laderas. Los agricultores se instalaron obviamente en las tierras más 
fértiles y llanas, donde se encontraban los Quercussp., que además fueron especialmente diezmados por el intenso aprovechamiento que han tenido debido a su alto valor como energía calorífica. Esto benefició la expansión de Pinus halepensis, que aprovechó las zonas de cultivo abandonadas y que ha sido potenciado por la repoblación forestal (Carrión y Dupré, 1996; Carcaillet et al., 1997; Mataix-Solera y Guerrero, 2007).

Hasta mediados del siglo XX, en España y el resto de países mediterráneos se garantizaba la gestión del monte mediante un aprovechamiento sistemático,y a veces incluso abusivo, de los recursos forestales del territorio. Los vecinos lo explotaban obteniendo leña para la lumbre y cocina, madera y pasto. Además se roturaba el suelo o se quemaba la vegetación para sembrar cereales, plantar frutales u olivos. El resultado era un paisaje compartimentado y diverso con bajo riesgo de incendios, los cuales eran rápidamente sofocados por una población que vivía en el monte y para el monte. No obstante, en algunas ocasiones tambien se producian incendios incontrolados como el de la Font Roja en Alcoy (Alicante) en 1840. Sin duda, el monte mediterráneo es el resultado de la explotación y el aprovechamiento humano (Montiel Molina, 1994; Vélez Muñoz, 1999;Molinero et al., 2008).

TABLA 1

ESPECIES ARBÓREAS MÁS AFECTADAS POR LOS INCENDIOS FORESTALES POR COMUNIDADES AUTÓNOMAS. DECENIO 1996-2005. (*) NAVARRA DE 2000-2005

\begin{tabular}{|l|l|r|l|r|}
\hline \multicolumn{1}{|c|}{ Comunidad autónoma } & \multicolumn{1}{|c|}{ Especie 1 } & Superficie (ha) & Especie 2 & Superficie (ha) \\
\hline Andalucía & Eucalyptus camaldulensis & 18372,39 & Pinus pinea & 10385,38 \\
\hline Aragón & Pinus halepensis & 2015,11 & Pinus sylvestris & 1455,17 \\
\hline Asturias & Eucalyptus globulus & 5874,72 & Pinus pinaster & 4108,28 \\
\hline Canarias & Pinus canariensis & 10261,83 & Pinus radiata & 52,03 \\
\hline Cantabria & Quercus pyrenaica & 1346,68 & Pinus sylvestris & 1181,78 \\
\hline Castilla La Mancha & Pinus pinaster & 13552,91 & Pinus halepensis & 8842,34 \\
\hline Castilla y León & Pinus pinaster & 18765,23 & Quercus pyrenaica & 15391,47 \\
\hline Cataluña & Pinus halepensis & 12073,61 & Pinus nigra & 11257,78 \\
\hline Comunidad Valenciana & Pinus halepensis & 9881,98 & Pinus nigra & 554,94 \\
\hline Extremadura & Pinus pinaster & 17887,52 & Quercus ilex & 7018,03 \\
\hline Galicia & Pinus pinaster & 42518,96 & Eucalyptus globulus & 21119,72 \\
\hline Islas Baleares & Pinus halepensis & 1232,64 & Quercus ilex & 11,55 \\
\hline La Rioja & Quercus pyrenaica & 47,48 & Pinus halepensis & 28,66 \\
\hline Madrid & Pinus pinea & 1320,93 & Pinus pinaster & 493,67 \\
\hline Murcia & Pinus halepensis & 661,70 & Quercus ilex & 8,76 \\
\hline Navarra (*) & Fagus sylvatica & 209,38 & Pinus halepensis & 205,58 \\
\hline País Vasco & Pinus radiata & 1743,54 & Eucalyptus globulus & 285,57 \\
\hline
\end{tabular}

Fuente: Ministerio de Medio Ambiente, 2006 
Pero en los años 60, la industrialización y el éxodo rural dieron lugar al abandono de los campos de zonas de montaña y contribuyeron a un incremento de la cubierta vegetal. En la actualidad, estas zonas no tienen apenas explotación ni se obtiene beneficio directo, ni siquiera para la gente que aún permanece en las zonas rurales, ya que nuevas normas y leyes restringen las talas, zonas de pasto y caza (Molinero et al., 2008). A este cambio de usos del suelo y al incremento del riesgo de incendios forestales han contribuido también las repoblaciones de pinar (la especie arbórea más afectadas por el fuego en Cataluña, Aragón, Comunidad Valenciana y Murcia es Pinus halepensis) (Tabla 1) y el aumento de nuevas zonas residenciales en la interfaz urbano-forestal (Pausas et al., 2008; Vallejo et al., 2009).

Las consecuencias han sido un aumento de los incendios forestales desde los años 70 en aquellos momentos en que se dan las condiciones climáticas favorables, a pesar del esfuerzo en pistas forestales, cortafuegos, infraestructuras y medios de extinción.El territorio valenciano es un ejemplo de esta relación entre abandono de las zonas interiores interior hacia el litoral y el incremento de los incendios forestales (Figura 1, 2) (Romero González, 1978; Pausas, 2004a). El fuego ocurre con mayor frecuenciay, aunque los ecosistemas mediterráneos son capaces de convivir con él, este cambio de régimen puede provocar efectos adversos. Pero los incendios forestales no son siempre eventos catastróficos (Mataix-Solera y Guerrero, 2007), losincendios de baja intensidad son de pequeño impacto y promueven la vegetación herbácea, incrementan la disponibilidad de nutrientes y aclaran los bosques, lo que promueve un hábitat más sano (Neary et al., 1999). Este fue el incendio controlado del matorral Mediterráneo durante milenios.

Figura 1

SUPERFICIE (MILES HA) AFECTADA POR INCENDIOS FORESTALES EN LA COMUNIDAD VALENCIANA, ESPAÑA Y OTROS PAISES DE EUROPA MEDITERRÁNEA (PORTUGAL, FRANCIA, ITALIA Y GRECIA)

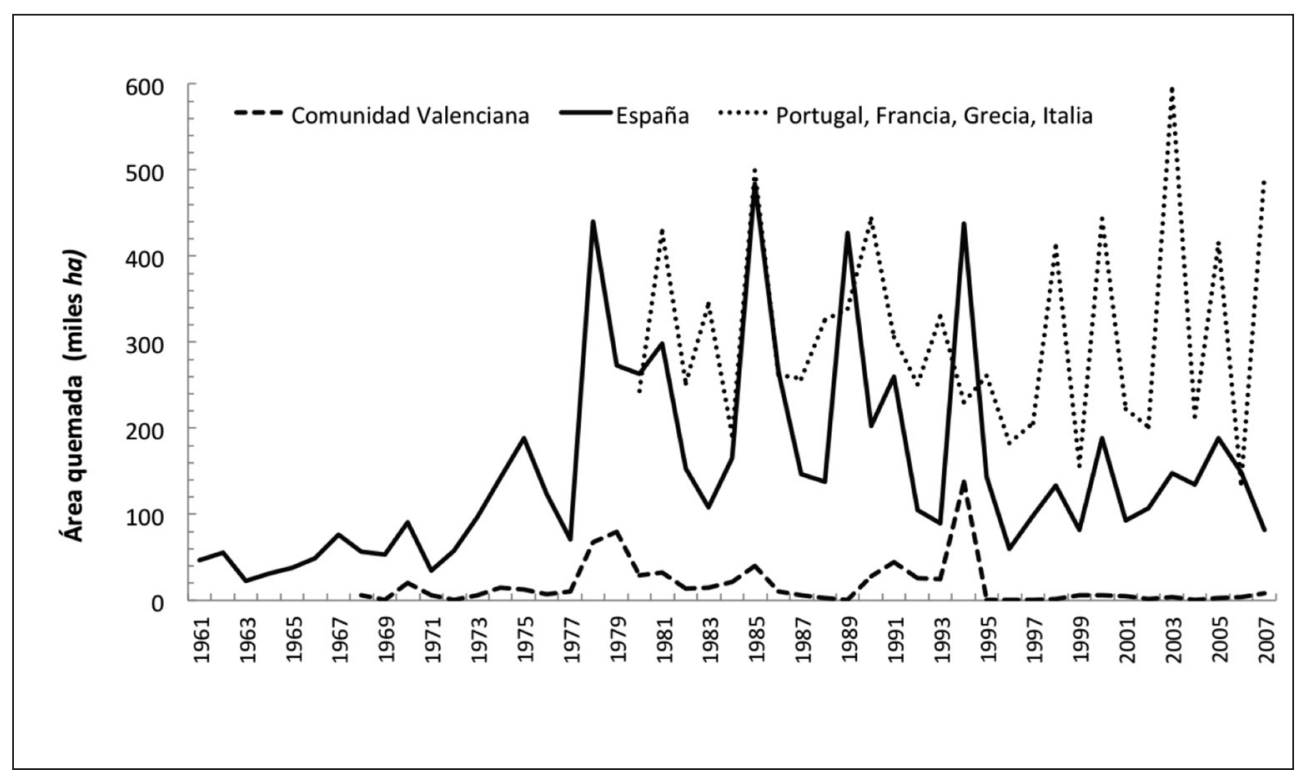

Fuente: Ministerio de Medio Ambiente, 2006; European Comission, 2006; Generalitat Valenciana, 2009. 


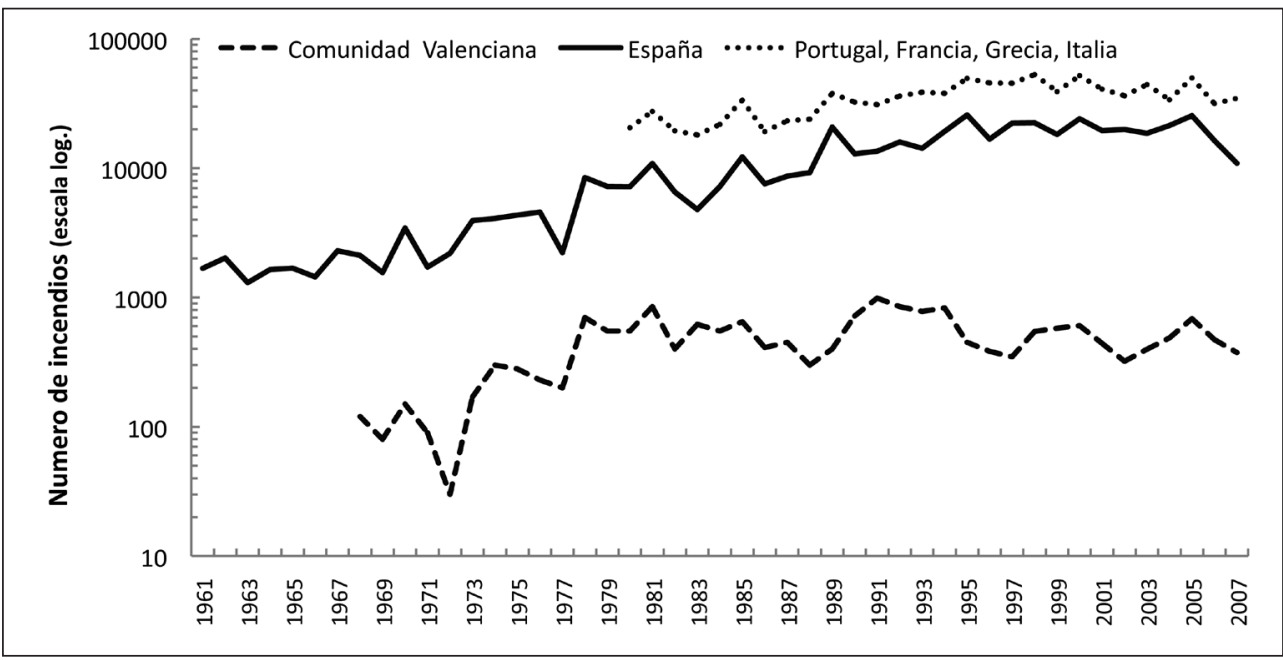

Fuente: Ministerio de Medio Ambiente, 2006; European Comission, 2006; Generalitat Valenciana, 2009.

\section{EFECTOS DE LOS INCENDIOS FORESTALES}

El fuego modifica los ciclos biogeoquímicos, produce cambios en la vegetación, suelo, fauna, procesos hidrológicos y geomorfológicos, calidad de las aguas e incluso cambios en la composición de la atmósfera (Prodon et al., 1987; DeBano et al., 1998; Shakesby y Doerr, 2006; Moody y Martin, 2009; Raison et al., 2009).

Los efectos de los incendios son muy variados debido a los múltiples factores de los que depende el incendio: biomasa disponible, intensidad (temperaturas alcanzadas y duración), área quemada, tiempo desde el último incendio, tipo de suelo, humedad, pendiente y vegetación (Neary et al., 1999). Así, se conforma en cada ecosistema un régimen de incendios concreto. Sin embargo, en un mismo ecosistema e incluso en un mismo incendio, la severidad, entendida como el grado de impacto en el ecosistema (Keeley, 2009), y efectos del fuego son diferentes y resultan en un mosaico de manchas de vegetación y suelo que se recuperará con o sin rehabilitación y restauración posterior (Figura 3). En esta recuperación, los efectos del fuego sobre la vegetación y los suelos son esenciales ya que influyen directamente sobre la evolución del resto del ecosistema.

\section{Efectos del fuego en la vegetación}

El aparente vacío biológico que queda tras un incendio es fugaz. Es frecuente observar una gran actividad biológica posterior y plantas simbiontes con algas tras las primeras lluvias (Mataix-Solera y Guerrero, 2007). Además, hay especies vegetales que presentan adaptacio- 
nes encaminadas a resistir y propagar el fuego, algunas incluso han desarrollado mecanismos reproductivos y ciclos vitales que dan ventaja a la especie cuando el fuego está presente. Todos estos signos denotan cierta compatibilidad y facilidad de recolonización (Arianoutsou et al., 1993).

Figura 3

INCENDIOS CON DIFERENTE SEVERIDAD INCENDIOS: A. INCENDIO EN PINOSO (ALICANTE) DONDE ALGUNOS DE LOS PINOS NO SE HAN QUEMADO COMPLETAMENTE, PERO CON MANCHAS DE CENIZAS BLANCAS QUE INDICAN LA TOTAL COMBUSTIÓN EN ALGUNAS ZONAS. B. INCENDIO EN LA SIERRA DE ENGUERA (VALENCIA), SE PUEDEN VER LOS PINOS COMPLETAMENTE QUEMADOS

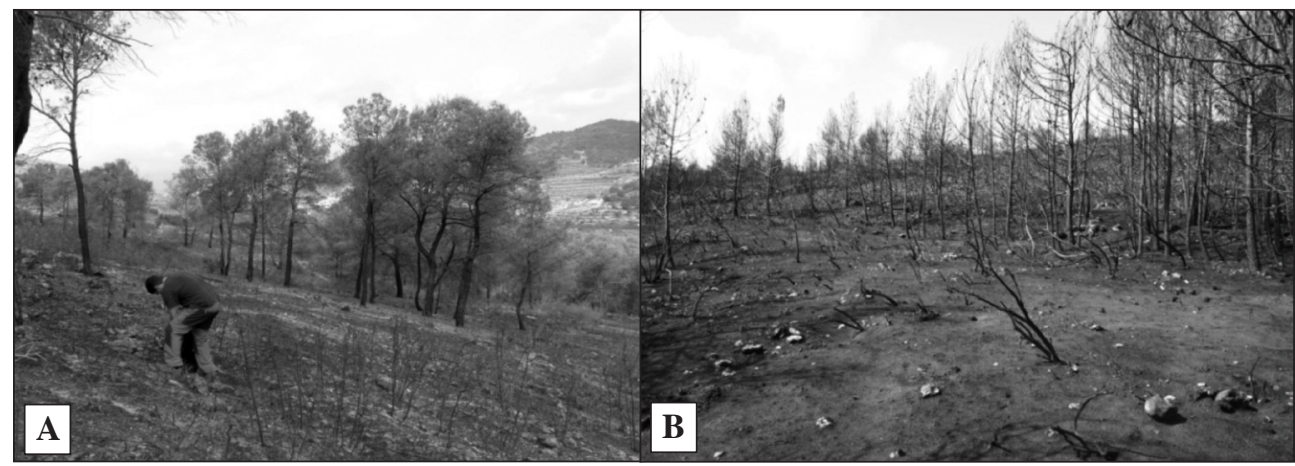

Fotografía de los autores.

Existen dos grandes grupos de especies vegetales según su respuesta al fuego: las especies rebrotadoras y las no rebrotadoras o germinadoras. Las primeras tienen la capacidad de rebrotar después de un incendio y en las segundas mueren los individuos pero no las semillas. También hay especies rebrotadoras que poseen además semillas adaptadas al fuego (especies facultativas) y finalmente hay especies que no pueden rebrotar, ni sus semillas resisten las altas temperaturas. Éstas desaparecen temporalmente después de un incendio y colonizan el espacio desde los extremos de la zona quemada (Pausas, 2004b; Lloret y Zedler, 2009).

El rebrote es uno de los mejores mecanismos de piroresistencia. Para ello se desarrollan cortezas gruesas y poco inflamables, que actúan como aislantes térmicos que protegen a la planta para que después del incendio sea capaz de rebrotar. En estos casos, las partes aéreas se queman pero se mantiene viva la cepa, que en algunos casos está constituida por un tejido llamado lignotuber que actúa como almacén de agua y nutrientes para asegurar la pervivencia de la planta (Molinas y Verdaguer, 1993). Cada rebrotadora dispone de distintas posibilidades de resistencia según la severidad del incendioy el momento de crecimiento de la planta. Los ejemplos son múltiples en la cuenca mediterránea: Quercus suber, con una magnifica corteza aislante (el corcho), Quercus coccifera, Quercus ilex, Juniperus Oxycedrus, Erica multiflora, Chamaerops humillis, así como en el chaparral californiano, el «fynbos» de Sudáfrica y los bosques australianos de «jarrah» (Arianoutsou et al., 1993)

Quercus coccifera es la especie protagonista durante la recuperación de la cubierta vegetal forestal en la cuenca mediterránea. Esta especie tiene una gran capacidad de rebrote inmediatamente después del incendio y un gran sistema radicular que permite en 2 ó 3 años un 
90\% de recubrimiento (Sala et al., 1990). Trabaud (1973) experimentó quemando esta especie reiterativamente con diferentes frecuencias (cada 6, 3 y 2 años) y en diferente estación durante 19 años y Quercus coccifera no tuvo ningún síntoma de degradación junto con Rubia peregrina y Brachypodium ramosum. No obstante, otros estudios apuntan que, en áreas más áridas al sur, Quercus coccifera puede tener un decrecimiento tras alternancias repetitivas (Delitti et al., 2005). Otras especies incrementan su población después de los incendios: Lonicera implexa (Trabaud, 1973), Phyllirea angustifolia, Erica arbórea (Moreno et al., 2004), plantas perennes como Ampelodesmos mauritanica (Lloret et al., 2003)y geófitas dotadas de bulbos Gladiolus, Asphodelus, Ophrys, Acis nicaeensis (Naveh, 1975; Diadema et $a l ., 2007)$. Pero por otra parte, hay especies que tienen tendencia a decrecer con el aumento de la recurrencia de incendios: Erica multiflora (Lloret et al., 2003), Rhammus alaternus y Smilax aspera (Trabaud, 1973). Sin embargo, en general parece ser que hay diferencias regionales en su habilidad rebrotadora (Pausas et al., 2008) (Figura 4, A, B, C, D).

Por otra parte, las plantas germinadoras adaptadas a los incendios retienen las semillas por largo tiempo hasta que son estimuladas por el calor y se dispersan. Son semillas serótinas. En estos casos, los individuos no resisten al incendio y son substituidos por otros que nacen de sus semillas y que encuentran un espacio sin competencia, donde llega mucha luz y el suelo es rico en nutrientes (DeBano et al., 1998; Pausas, 2004b). Buenos ejemplos son las piñas que se abren con el calor y dispersan los piñones, en especies como Pinus halepensis, Pinus brutia, Pinus pinaster, permitiendo una rápida regeneración del pinar (Arianoutsou et $a l ., 1993)$. Otros pinos con piñas no serótinas como el Pinus pinea, tienen piñones muy duros que resisten a los incendios. Pero hay otras especies de pinos muy sensibles (Pinus nigra, Pinus sylvestris) que pueden ser eliminados temporalmente según la extensión del incendio (Pausas et al., 2008). Existen arbustos no rebrotadores que tienen bancos de semillas persistentes en el suelo y resisten el calor del fuego, como las Cistus sp. o Ulex parviflorus (que además acumula mucha necromasa en su parte aérea y cuando llega el fuego es altamente combustible). Hay otras especies en que las semillas son estimuladas para germinar por otros productos derivados del incendio como el humo o las cenizas (por ejemplo: Rhamnus alaternus, Alnus glutinosa, Cistus incanus, Clematis vitalba) (Crosti et al., 2006; Paula et al., 2006; Reyes y Casal, 2006). Sin embargo, si el periodo entre incendios no es suficiente para que los individuos lleguen a la edad adulta, o si este intervalo corto entre incendios es muy recurrente, el banco de semillas se puede agotar (Pausas, 2004b).

Después de un incendio no hay sucesión en el sentido de que unas comunidades reemplazan a otras, sino que hay una progresiva reaparición de las especies que pertenecían ya a la comunidad (Trabaud, 1990). Tras el incendio, las herbáceas de vida corta cubren el suelo junto con los arbustos y leñosas que rebrotan rápidamente. Las herbáceas alcanzan su pico en 1-5 años, y luego reducen su presencia y diversidad. Hay algunas para las que el fuego es esencial, ya que sólo aparecen tras el incendio al estar sus semillas en estado latente (De Lillis y Testi, 1990). Los rebrotes de leñosas son siempre muy rápidos debido al sistema raticular bien desarrollado del que disponen, lo que permite captar agua y nutrientes, aunque también las no rebrotadoras como Cistus sp., Ulex parviflorus y Rosmarinus officinalis aparecen pronto. El crecimiento es muy activo en el primer quinquenio y luego se ralentiza hasta ser casi nulo 25 ó 30 años después del incendio (Ferran y Vallejo, 1992; Arianoutsou et al., 1993; Ferran y Vallejo, 1998). En este aspecto, las variaciones son grandes y dependen 
de las características del incendio y del lugar donde se produzca (Tabla 2). Esta rapidísima regeneración es muy importante para proteger el suelo de la erosión.

Se ha comprobado que las comunidades vegetales de los ecosistemas mediterráneos tienen en general una alta resiliencia a los incendios forestales, es decir tienen gran habilidad para volver a las condiciones anteriores a la alteración (Lloret y Zedler, 2009). La vegetación resistente al fuego ha evolucionado hasta conseguir un mantenimiento y regeneración con un determinado régimen de incendios. Cuando un régimen de incendios permanece en un ecosistema por largo tiempo se produce un tipo de relación entre la resiliencia de las especies y el régimen de incendios (Lloret y Zedler, 2009). Según Naveh (1990), para estas especies el fuego puede que sea la única manera de asegurar el rejuvenecimiento. Pero no hay que olvidar que las especies están adaptadas a un régimen de incendios, no a cualquier incendio, y el cambio de régimen de incendios puede tener impactos importantes en la sostenibilidad de algunos de los componentes (Pausas y Keeley, 2009).

Figura 4

ESPECIES REBROTADORAS 6 MESES DESPUÉS DE UN INCENDIO EN LA SIERRA DE ENGUERA (VALENCIA), A FINALES DE ABRIL DE 2008. A. QUERCUS COCCIFERA, UNA DE LAS PRIMERAS EN REBROTAR. B. JUNIPERUS OXYCEDRUS Y RHAMNUS LYCIOIDES.C. ERICA MULTIFLORA. D. QUERCUS ILEX

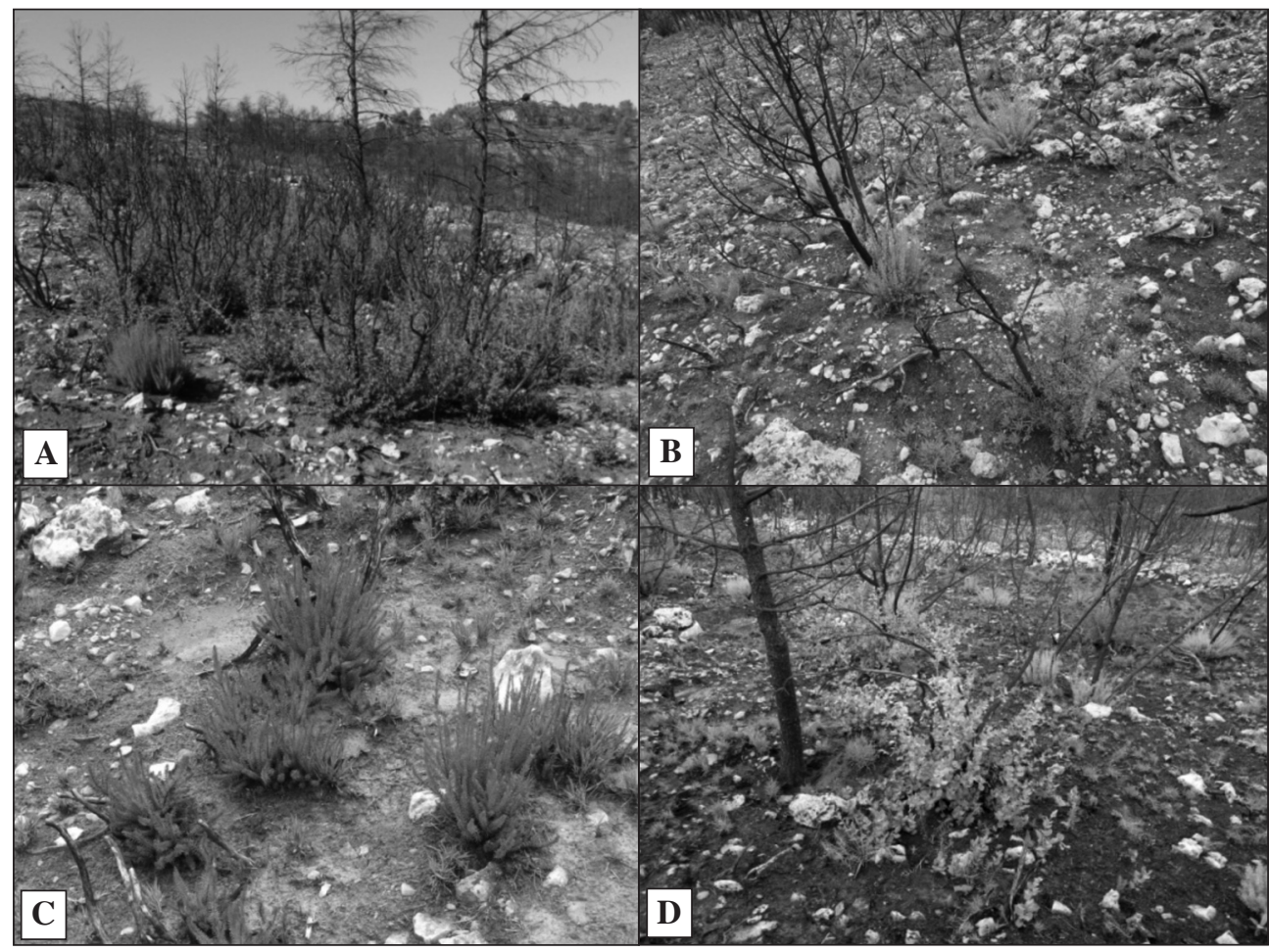

Fotografía de los autores. 


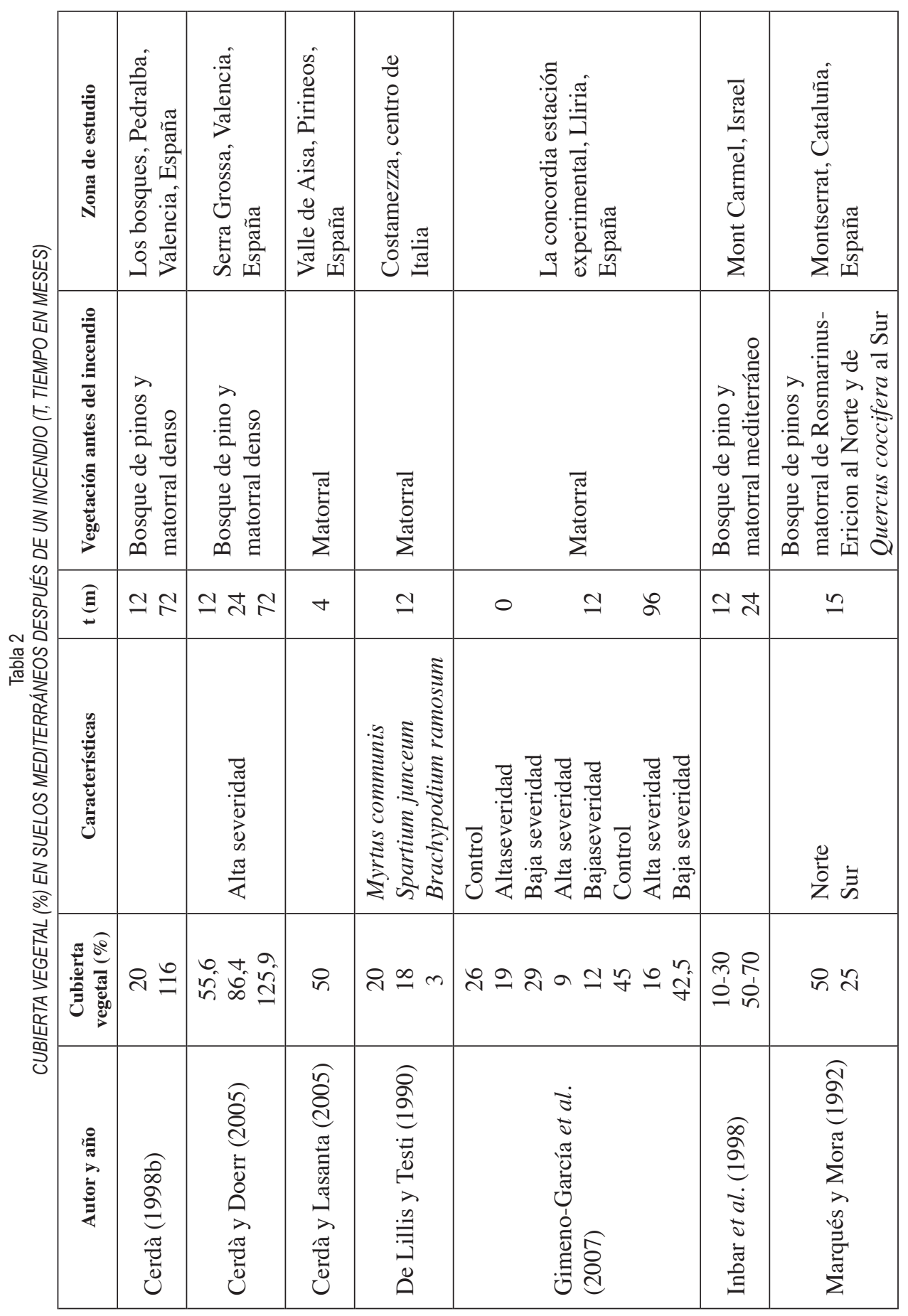




\begin{tabular}{|c|c|c|c|}
\hline 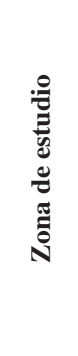 & 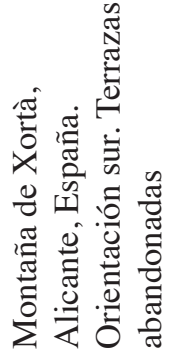 & 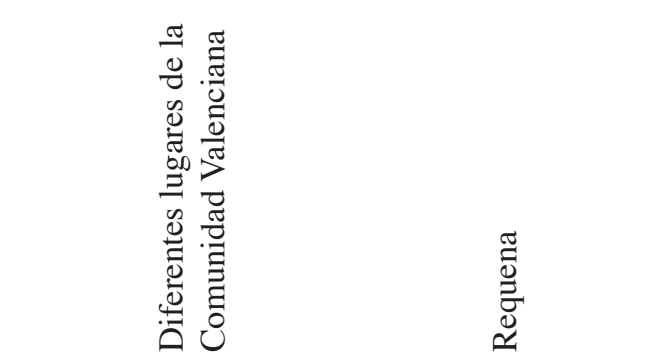 & 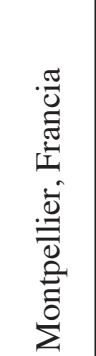 \\
\hline 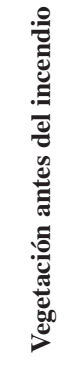 & 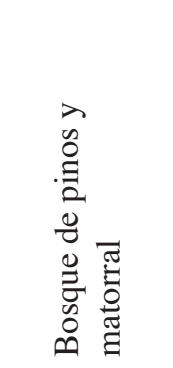 & 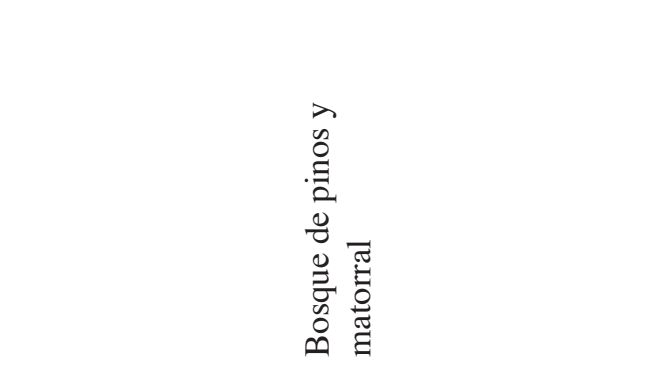 & 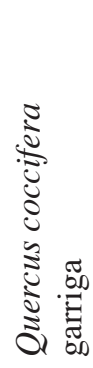 \\
\hline$\widehat{\Xi \Xi}$ & $\underset{+}{+} N$ & 으의 & $\simeq$ \\
\hline 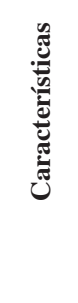 & 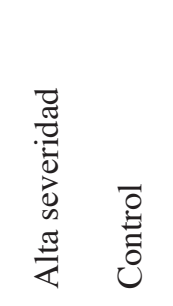 & 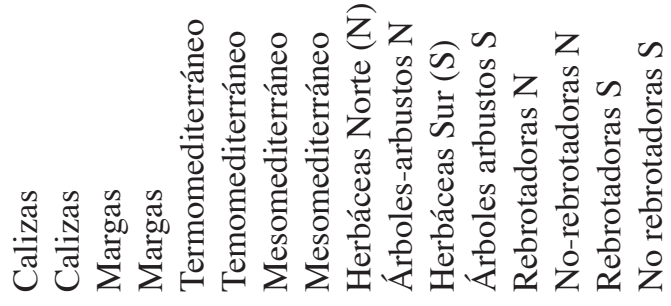 & 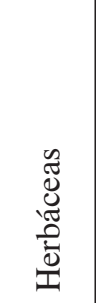 \\
\hline 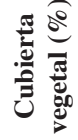 & ํำ & 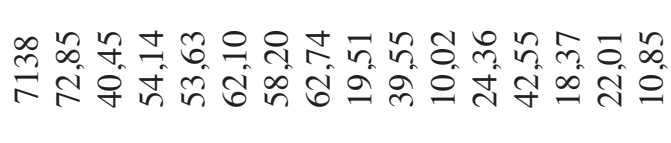 & 유 \\
\hline 高 & 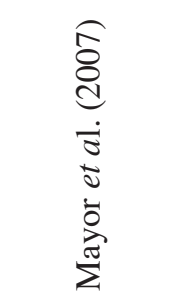 & 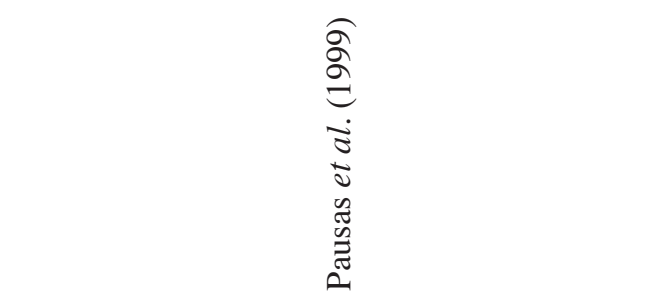 & 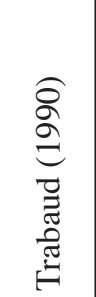 \\
\hline
\end{tabular}




\section{Efectos del fuego en el suelo}

El suelo es el componente básico del ecosistema forestal. Su sostenibilidad y recuperación dependen de las funciones y procesos químicos, físicos y biológicos que ocurran debajo de la capa de hojarasca (Neary et al., 1999; Mataix-Solera y Guerrero, 2007). Tras el fuego, el suelo puede sufrir cambios directos producidos por el calentamiento y la combustión, e indirectos como consecuencia de la situación microclimática después de la pérdida de la cubierta vegetal y recubrimiento de las cenizas (Figura 5, A). Estos cambios dependerán principalmente de la temperatura alcanzada durante el incendio (Neary et al., 1999).

El calentamiento del suelo produce variaciones en algunas de las propiedades físicas y químicas. El pH y la conductividad eléctrica normalmente aumentan, debido al aporte de carbonatos, cationes básicos y óxidos procedentes de las cenizas. El tiempo de recuperación del pH inicial es variado y se considera que es más o menos rápido según el tiempo que las cenizas permanezcan en el suelo (Mataix-Solera y Guerrero, 2007). Este aporte de cenizas también enriquece el suelo con un aumento de nutrientes ( $\mathrm{Ca}, \mathrm{Mg}, \mathrm{K}, \mathrm{Na}, \mathrm{P}$ ) y según Kutiel y Naveh (1987b) es considerado el mayor factor de crecimiento de la vegetación en los ecosistemas mediterráneos. Sin embargo hay algunos nutrientes que se pierden con el humo del fuego, se volatilizan (Raison et al., 1984), o existe peligro de que la acción del viento, erosión o lixiviación laven esta inyección de nutrientes fundamentales, sobre todo cuando no hay vegetación (Arianoutsou et al., 1993; Neary et al., 1999; Cerdà y Bodí, 2007). Así, esta fertilización puede ser efímera, durar 4-5 meses (Kutiel y Naveh, 1990; Gimeno-García et al., 2000), hasta 14 meses (Kutiel y Naveh, 1987b) o incluso 7 años (Úbeda et al., 2005).

El nitrógeno es uno de los elementos que más fácilmente se volatiliza. Durante la combustión se pueden perder grandes cantidades, pero afortunadamente para los ecosistemas mediterráneos y regiones semiáridas donde es limitado, se suele encontrar tras el incendio más nitrógeno disponible en el suelo en la forma de amonio $\left(\mathrm{NH}_{4}{ }^{+}-\mathrm{N}\right)(\mathrm{Kutiel}$ y Naveh, 1987a; Giovannini et al., 1990b; Gimeno-García et al., 2000). Asimismo, en los meses posteriores se produce un aumento del nitrógeno $\left(\mathrm{N}_{2}\right)$ por microorganismos promovidos por el incendio y especies de leguminosas fijadoras como el Ulex parviflorus (Arianoutsou et al., 1993; Pastor-López y Martin-Martin, 1995; Neary et al., 1999; Raison et al., 2009).

Respecto a los cambios en el carbono orgánico del suelo, los resultados son complejos y variados según la intensidad del incendio. En incendios de baja intensidad puede haber incrementos de carbono orgánico procedente de la vegetación parcialmente pirolizada, en cambio, a intensidades elevadas la cantidad de materia orgánica de la superficie del suelo puede disminuir (Mataix-Solera et al., 2002). Según Knoepp et al. (2005) calentando el suelo a $450{ }^{\circ} \mathrm{C}$ durante dos horas o a $500{ }^{\circ} \mathrm{C}$ media hora, se destruye el $99 \%$ de la materia orgánica. Pero el fuego no sólo modifica la cantidad de la materia orgánica, también altera su calidad. Actúa como un agente que acelera las tasas de mineralización del carbono orgánico y además modifica las tasas de descomposición postincendio ya que, a medida que se incrementa la temperatura, el humus sufre modificaciones que le hacen más resistentes a la degradación microbiana (González-Vila et al., 2009; Knoepp et al., 2005). Esta materia orgánica carbonizada que se produce en grandes cantidades y se acumula en el suelo, puede contribuir en un $30-40 \%$ al carbono del suelo en ecosistemas propensos a incendios forestales y al secuestro 
de carbono a largo plazo, siendo un componente significativo en el ciclo global del carbono (Forbes et al., 2006).

La estabilidad de los agregados también puede cambiar tras el paso del fuego. Generalmente se detecta una reducción de la estabilidad de los agregados relacionado con la pérdida de materia orgánica (Cerdà, 1993; DeBano et al., 1998; Badia y Martí, 2003). No obstante, es posible encontrar tendencias contrarias cuando hay incendios de baja intensidad en los que aumenta la materia orgánica (Díaz-Fierros et al., 1987), debido a fusiones de arcillas por calcinación que endurecen los agregados (Giovannini et al., 1990a) o incluso debido la cementación por las sustancias hidrofóbicas que los hacen más resistentes (Giovannini y Lucchesi, 1983). La porosidad y la capacidad de retención hídrica también pueden verse disminuidas al cambiar la estructura del suelo y desaparecer la materia orgánica si las intensidades son más elevadas (Neary et al., 1999). Estos tres factores, junto con la hidrofobicidad, son aspectos fundamentales que determinaran la aireación, infiltración y erodibilidad de un suelo, sobre todo cuando se elimina la vegetación y hojarasca.

Figura 5

A. CAPA DE CENIZAS QUE CUBRE EL SUELO DESPUÉS DE UN INCENDIO EN PINOSO (ALICANTE) EN JULIO DE 2009. B. HIDROFOBICIDAD EN UN SUELO QUEMADO EN LA SIERRA DE ENGUERA (VALENCIA) EN 2008. EL MÉTODO UTILIZADO ES ELTEST DE PENETRACIÓN DE LA GOTA DE AGUA (WDPT) QUE CONSISTE EN APLICAR GOTAS DE AGUA DESTILADA SOBRE EL SUELO Y MIDIENDO EL TIEMPO QUE TARDAN EN INFILTRARSE

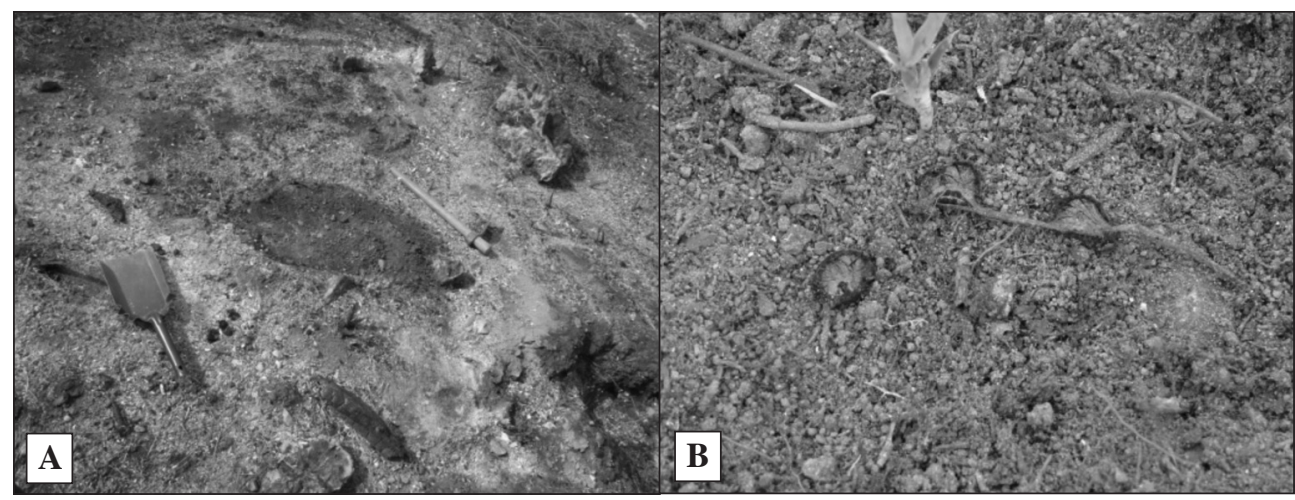

Fotografía de los autores.

La hidrofobicidad o repelencia al agua puede verse provocada, aumentada o disminuida según la temperatura alcanzada en el suelo. A grandes rasgos, si la temperatura alcanzada en el suelo es de $200-250^{\circ} \mathrm{C}$, la provoca o la aumenta, y si es mayor de $300^{\circ} \mathrm{C}$, la destruye (DeBano, 1981). Ésta se debe a la existencia de sustancias orgánicas que se volatilizan durante la combustión y se condensan posteriormente (Doerr et al., 2000). Los suelos ácidos y de textura arenosa son más propensos a la hidrofobicidad (Mataix-Solera y Doerr, 2004), pero ésta también aparece en suelos calcáreos (Figura 5, B) (Cammeraat y Imeson, 1999; Cerdà y Doerr, 2005; Arcenegui et al., 2007). No obstante, se ha comprobado que las mismas propiedades del suelo pueden controlar si el suelo desarrolla hidrofobicidad como consecuencia de la combustión. Mataix-Solera et al. (2008) demostró que la baja 
ratio materia orgánica/arcilla y una presencia elevada de caolinita en el suelo denominado comúnmente como terra rossa (principalmente Rhodoxeralfs), lo hacen muy poco susceptible a desarrollar esta propiedad. En los demás suelos mediterráneos calcáreos, los factores que principalmente controlan la hidrofobicidad son, junto con la temperatura alcanzada, el tipo de vegetación y la cantidad de hojarasca presente, siendo Pinus halepensis y Rosmarinus officinalis los mayores inductores de sustancias hidrofóbicas en el suelo (Arcenegui et al., 2007; Tessler et al., 2008). Sin embargo, la repelencia al agua ocurre también en suelos no afectados por incendios forestales y depende principalmente del tipo de vegetación presente y de la humedad del suelo (Mataix-Solera et al., 2007; Doerr y Shakesby, 2009). La persistencia y la distribución espacial de la hidrofobicidad es un factor clave en la dinámica de las escorrentías en los suelos quemados. Como consecuencia inmediata, se produce una reducción de la infiltración y un aumento de escorrentía y la erosión, además de disminuir la humedad del suelo en la zona hidrófoba (Doerr et al., 2009).

Los efectos indirectos del fuego sobre los suelos se producen a partir de la desaparición de la cubierta vegetal, la adición de cenizas y el ennegrecimiento del suelo. Estas modificaciones suponen cambios microclimáticos en la humedad edáfica, temperatura y radiación solar (Raison et al., 2009) que afectarán a la recuperación del sistema tanto en beneficio (menos competencia, más luz, más nutrientes) como en detrimento (mayor erosión, menos infiltración) del ecosistema (Neary et al., 1999).

La hidrología del suelo se modifica como consecuencia del aumento de la hidrofobicidad, reducción de la materia orgánica, disminución de la porosidad y estabilidad de los agregados, el sellado del suelo por partículas minerales o cenizas y sobre todo la reducción de la cubierta vegetal (MacDonald et al., 2008). 15 MM EN 10 MINUTOS DOS MESES Y MEDIO DESPUÉS DEL INCENDIO

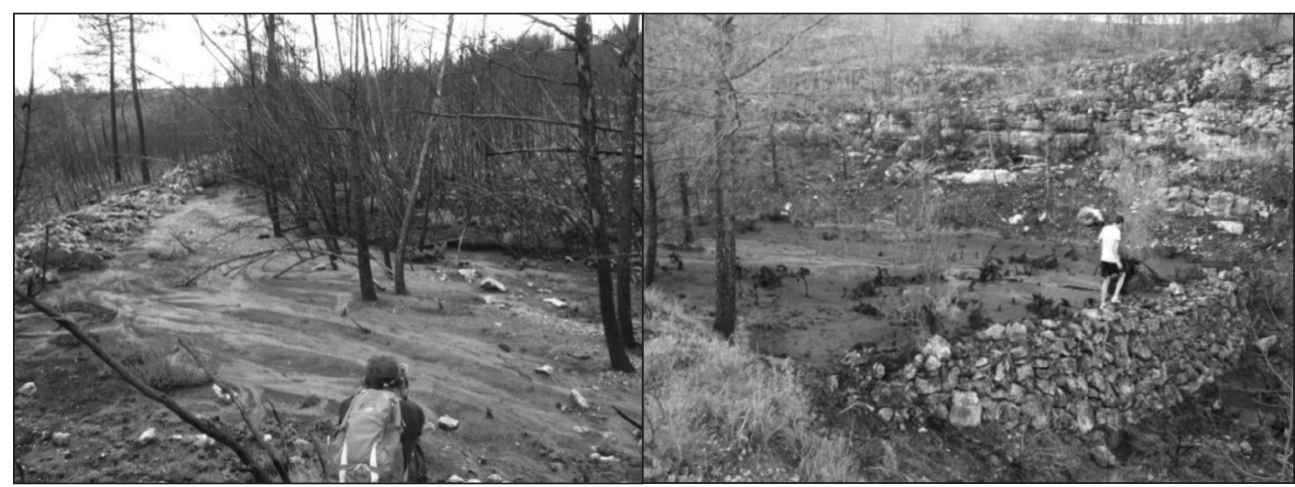

Fotografía de los autores.

Inmediatamente después del incendio las tasas de escorrentía son prácticamente nulas, debido a la capa de cenizas que cubre el suelo (Cerdà, 1998b; Cerdà y Doerr, 2008). Más tarde, tras la pérdida de las cenizas o su encostramiento, se produce un aumento sustancial de las tasas de escorrentía y erosión que se van reduciendo con la recuperación de la vegetación. 
No obstante, las magnitudes tanto de las tasas de escorrentía como de erosión son variadas (ver revisión de Cerdà y Bodí, 2007) y las tasas de erosión no suelen ser superiores a $1 \mathrm{Mg}$ $\mathrm{ha}^{-1}$ año $^{-1} \mathrm{y}$ por tanto se encuentran dentro del rango de sostenibilidad y tolerancia (Cerdà y Bodí, 2007). Las tormentas individuales de gran intensidad son las que desencadenan los episodios extraordinarios de arroyada post-incendio (Figura 6). Durante estas tormentas, las cuencas quemadas generalmente responden más rápido a la lluvia que antes del incendio o que cuencas no quemadas. En las zonas mediterráneas tras el incendio de verano, el suelo está desprovisto de vegetación y queda más expuesto al poder erosivo de las lluvias torrenciales otoñales (Marqués y Mora, 1992; Shakesby y Doerr, 2006; Mayor, 2007).

La recuperación de las tasas de erosión previas al incendio es variable y en ocasiones contrastada (ver Cerdà y Lasanta, 2005). En diversos trabajos se ha comprobado que las tasas de escorrentía y erosión se recuperan a los 2 años (Marqués y Mora, 1992; Cerdà, 1998a; Cerdà y Doerr, 2005), aunque los casos habría que matizarlos. Para Cerdà (1998b), las tasas de escorrentía se igualan con las iniciales después de 4 años para las condiciones de invierno, pero 2 en verano debido a las mayores tasas de infiltración con suelos secos. En zonas más áridas, la recuperación puede durar 3-4 años (May, 1990), 6 años (Mayor et al., 2007) u 8, si la vegetación no se regenera rápidamente (Gimeno-García et al., 2007). Incluso a microescala, en zonas abruptas con piedras y vegetación quemada, la vuelta a la situación de antes del incendio se produce al cabo de un año a diferencia de las zonas llanas, donde sigue siendo alta porque hay más suelo que erosionar (Lavee et al., 1995). La regeneración de la vegetación se considera el factor más importante para reducir la erosión en el mediterráneo (Dieckmann et al., 1992; Cerdà, 1998a; Inbar et al., 1998).

Está claro que a la gran variabilidad espacial en los efectos de los incendios forestales se ha de sumar la variabilidad climática, orográfica, litológica y de usos del suelo que provocan que las respuestas a los incendios sean variadas (Cerdà y Bodí, 2007).

\section{IMPLICACIONES PARA LA GESTIÓN}

Como se ha comprobado a través de esta revisión bibliográfica, los ecosistemas mediterráneos son dependientes de los incendios en su debido régimen de recurrencia e intensidad (Bond et al., 2004). Aunque el fuego es una alteración, el ecosistema está preparado para volver a la situación inicial anterior al incendio. No obstante, la política forestal tradicional en la cuenca mediterránea ha replantado durante muchos años bosques monoespecíficos de pino después de los incendios, siendo algunos muy inflamables (Pausas et al., 2008). En otras ocasiones hahabido intentos de introducir semillas de Quercus sp., que normalmente tienen alta mortalidad (Beyers, 2009). Además, estas plantaciones se realizan mediante maquinaria pesada que produce una alteración dramática de los suelos y la vegetación. Así, la decisión de dónde y cuándo usar tratamientos de remediación después de un incendio requiere una evaluación de la severidad de incendio, el clima, suelos, topografía e hidrología de la cuenca (Robichaud, 2009). Las actuaciones post-incendio que se utilizan y están siendo estudiadas principalmente son el acolchado con restos vegetales e hidrosiembras, repoblaciones con diferentes tipos de semillas y barreras de erosión. No obstante, estos tratamientos post-incendio son muy caros y solo deben ser aplicados si el riesgo de degradación del suelo y vegetación es elevado. Por ello, es justificable y efectiva la no actuación post-incendio, tanto por 
razones económicas como medioambientales. Un buen ejemplo de ello lo encontramos en los casos en los que las hojas caídas de los pinos después de un incendio actúan como acolchado natural y la propia regeneración de la vegetación es suficiente para reducir las tasas de erosión (Cerdà y Robichaud coord., 2009). En caso de realizar una repoblación, la ejecución de ésta puede provocar más daño al suelo que el propio incendio, y obviamente es más caro que dejar que la vegetación se recupere por ella misma. Es necesario por tanto estudiar y analizar cada caso concreto para poder decidir si es mejor o no actuar.

Hasta ahora, las políticas forestales han optado por una estricta supresión de los incendios forestales y divulgan sólo sus efectos negativos, de manera que la sociedad ha llegado a considerar que el fuego debe suprimirse completamente. Pero, como se ha comprobado, esta política propicia los incendios de alta intensidad, precisamente los que desencadenan procesos de degradación más intensos. Ejemplos son las olas de grandes incendios como los de 1979 y 1994 en la Comunidad Valenciana y, más recientemente las de Galicia en 2006, Grecia en 2007 o el sureste de Australia en Febrero de 2009, lo que demuestra que no podemos excluir el fuego de unos ecosistemas que han convivido con él de manera natural y bajo la gestión del hombre. Es por tanto necesaria una gestión del monte que puede incluir desbroces o incluso en la que se contemple la posibilidad de usar el fuego controlado para combatir los incendios, opción que ya se aplica en Canarias y Cataluña y en países como EEUU o Australia. Además, esta fue la estrategia usada en el Mediterráneo durante milenios para abrir claros, evitar incendios no controlables, o producir pastos.

\section{CONCLUSIONES}

El fuego es un factor ecológico natural en los ecosistemas mediterráneos y ha contribuido a modelar el paisaje que conocemos, no solo por su recurrencia natural sino también por el uso que el ser humano ha hecho de éste. La investigación científica de los últimos 20 años ha corroborado esta visión, por lo que se acepta que el fuego es necesario para un correcto funcionamiento del ecosistema en los bosques mediterráneos. Sin embargo, el régimen de incendios ha cambiado debido a las nuevas tendencias sociales, económicas y por las políticas forestales de supresión del fuego. El reto por lo tanto es gestionar un régimen de incendios adecuado para las demandas sociales presentes y futuras evitando el riesgo que suponen los incendios y a la vez conseguir la sostenibilidad del ecosistema.

\section{AGRADECIMIENTOS}

Se agradece a Joan Romero,Alejandro Pérez Cueva y a dos revisores anónimos su revisión y comentarios. Este trabajo no habría sido posible sin la financiación de la beca FPU del Ministerio de Ciencia e Innovación.

\section{BIBLIOGRAFÍA}

ARCENEGUI, V., MATAIX-SOLERA, J., GUERRERO, C., ZORNOZA, R., MAYORAL, A. M. y MORALES, J. (2007): «Factors controlling the water repellency induced by fire in calcareous Mediterranean forest soils». European Journal of Soil Science, n 58, 1254-1259. 
ARIANOUTSOU, M., BEARD, J. S., FERRÉS, L., FOLCH, R. y TRABAUD, L. V. (1993): «La vida a les formacions escleròfil·les», en Mediterrànies (Folch, R. coord.). Barcelona, Edit. MAB, UNESCO, 64-109

BADIA, D. y MARTÍ, C. (2003): «Plant ash and heat intensity effects on chemical and physical properties of two contrasting soils». Arid Land Research and Management, $\mathrm{n}^{\circ}$ 17, 23-41.

BEYERS, J. L. (2009): «Non-native and native seeding», en Fire effects on soils and restoration strategies (Cerdà, A. y Robichaud, P. R. coord.). Enfield, Edit. Science Publishers, 321-336.

BODÍ, M. B., DOERR, S. H., MATAIX-SOLERA, J. y CERDÀ, A. (2008): «El papel del fuego en los ecosistemas terrestres». V Congreso «La investigación ante la sociedad del conocimiento». Sostenibilidad y Medio Ambiente. Escuela politécnica superior de Alcoi, UPV, 331-335.

BOND, W. J., WOODWARD, F. I. y MIDGLEY, G. F. (2004): «The global distribution of ecosystems in a world without fire». New Phytologist, $\mathrm{n}^{\circ} 165,525-538$.

BOND, W. J. y KEELEY, J. E. (2005): «Fire as a global «herbivore»: the ecology and evolution of flammable ecosystems». Trends in Ecology and Evolution, n ${ }^{\circ}$ 20, 387-394.

CAMMERAAT, L. H. y IMESON, A. C. (1999): «The evolution and significance of soilvegetation patterns following land abandonment and fire in Spain». Catena, $\mathrm{n}^{\circ}$ 37, $107-$ 127.

CARCAILLET, C., ALMQUIMIST, H., ASNONG, H., BRADSHAW, R. H. W., CARRIÓM, J. S., GAILLARD, M. J., GAJEWSKI, K., HAAS, J. N., HABERLE, S. G., HADOM, P., MULLER, S. D., RICHARD, P. J. H., RICHOZ, I., ROSCH, M., SANCHEZ GONI, M. F., VON STEDINGK, H., STEVENSON, A. C., TALON, B., TARDY, C., TINNER, W., TRYTERUD, E., WICK, L. y WILLIS, K. J. (2002): «Holocene biomass burning and global dynamics of the carbon cycle». Chemosphere, $\mathrm{n}^{\circ} 49,845-863$.

CARCAILLET, C., BARAKAT, H. N., PANAIOTIS, C. y LOISEL, R. (1997): «Fire and Late-Holocene expansion of Quercus ilex and Pinus pinaster on Corsica». Journal of Vegetation Science, $\mathrm{n}^{\circ} 8,85-94$.

CARRIÓN, J. S. y DUPRÉ, M. (1996): «Late Quaternary vegetation history at Navarrés, Eastern Spain. A two core approach». New Phytologist, n 134, 177-191.

CERDÀ, A. (1993): «Incendios forestales y estabilidad de los agregados». Cuadernos de Geografía, $\mathrm{n}^{\circ}$ 53, 1-16.

CERDÀ, A. (1998a): «Post-fire dynamics of erosional processes under Mediterranean climatic conditions». Z. Geomorph.N.F., no 42, 373-398.

CERDÀ, A. (1998b): «Changes in overland flow and infiltration after a rangeland fire in a Mediterranean scrubland». Hydrological Processes, $\mathrm{n}^{\circ} 12,1031-1042$.

CERDÀ, A. y DOERR, S. H. (2005): «Influence of vegetation recovery on soil hydrology and erodibility following fire: an 11-year investigation». International Journal of Wildland Fire, $\mathrm{n}^{\circ} 14,423-437$.

CERDÀ, A. y BODÍ, M. B. (2007): «Erosión hídrica en suelos afectados por incendios forestales», en Incendios forestales, suelos y erosión hídrica, (Mataix-Solera, J. coord.). Alcoi, Edit. Caja de Ahorros del Mediterráneo CEMACAM, 71-118. 
CERDÀ, A. y DOERR, S. H. (2008): «The effect of ash and needle cover on surface runoff and erosion in the immediate post-fire period». Catena, $\mathrm{n}^{\circ} 74,256-263$.

CERDÀ, A., y JORDÁN, A., coord. (2010): Actualización en métodos y técnicas para el estudio de los suelos afectados por incendios forestales. Valencia, Cátedra de Divulgación de la Ciencia. Universitat de València.

CERDÀ, A., y LASANTA, T. (2005): «Long-term erosional responses after fire in the Central Spanish Pyrenees 1. Water and sediment yield». Catena, $\mathrm{n}^{\circ}$ 60, 59-80.

CERDÀ, A. y MATAIX-SOLERA, J., coord. (2009): Efectos de los incendios forestales sobre los suelos en España. El estado de la cuestión visto por los científicos españoles. Valencia, Cátedra Divulgación de la Ciencia, Universitat de València.

CERDÀ, A. y ROBICHAUD, P. R., coord. (2009): Fire effects on soils and restoration strategies. Enfield, NH, USA. Science Publishers.

European Commission, Directorate-General Joint Research Centre, Directorate-General Environment, Institute for Environment and Sustainability and Land Management y Natural Hazards Unit European Comission (2006): Forest Fires in Europe 2005. Report No 6. Italy.

CROSTI, R., LADD, P. G., DIXON, K. W. y PIOTTO, B. (2006): «Post-fire germination: the effect of smoke on seeds of selected species from the central Mediterranean basin». Forest Ecology and Management, $\mathrm{n}^{\circ} 221,306-312$.

DE LILLIS, M. y TESTI, A. (1990): «Post-fire dynamics in a disturbed Mediterranean community in central Italy» en Fire in ecosystem dynamics: Mediterranean and northern perspectives (Goldammer, J. G. y Jenkins, M. J. coord.). The Hague, Edit. SPB Academic Publishing, 53-62.

DEBANO, L. F. (1981): Water repellent soils: a state-of-the-art, edited by United States Department of Agriculture, F. S., p. 21, Pacific Southwest Forest and Range Experiment Station, Berkeley (California).

DEBANO, L. F., NEARY, D. G. y FFOLLIOTT, P. F. (1998): Fire's effects on ecosystems. New York. John Wiley and Sons.

DELITTI, W., FERRAN, A., TRABAUD, L. V. y VALLEJO, R. (2005): «Effects of fire recurrence in Quercus coccifera L. shrublands of the Valencia Region (Spain): I. plant composition and productivity». Plant Ecology, $\mathrm{n}^{\circ}$ 177, 57-70.

DIADEMA, K., MEDAIL, F. y BRETAGNOLLE, F. (2007): «Fire as a control agent of demographic structure and plant performance of a rare Mediterranean endemic geophyte». Ecology/Ecologie, $\mathrm{n}^{\circ} 330,691-700$.

DÍAZ-FIERROS, F., BENITO, E. y PÉREZ, R. (1987): «Evaluation of the U.S.L.E.: for prediction of erosion in burnt forest areas in Galicia (NW Spain)». Catena, $\mathrm{n}^{\circ}$ 14, 189-199.

DIECKMANN, H., MOTZER, H., HARRES, H. P. y SEUFFERT, O. (1992): «Vegetation and erosion. Investigation on erosion plots in Southern Sardinia». Geooko-plus, $\mathrm{n}^{\circ} 3$, 139-149.

DOERR, S. H., SHAKESBY, R. A. y WALSH, R. P. D. (2000): «Soil Water repellency: Its causes, characteristics and hydro-geomorphological significance». Earth Science Reviews, ${ }^{\circ}$ 51, 33-65.

DOERR, S. H. y SHAKESBY, R. A. (2009): «Soil Water repellency. Principles, causes and relevance in fire-affected environments», en Efectos de los incendios forestales sobre los 
suelos en España. El estado de la cuestión visto por los científicos españoles (Cerdà, A. y Mataix-Solera J. coord.). Valencia, Cátedra de divulgación de la ciencia, Universitat de València, 57-75.

DUPRÉ, M. (1983): «Los montes: su estado y política forestal en la cuenca del Júcar. Papel hidrológico-forestal». Cuadernos de Geografía, n 32-33, 265-290.

FERRAN, A. y VALLEJO, R. (1992): «Litter dynamics in post-fire successional forests of Quercus ilex». Vegetatio, $\mathrm{n}^{\circ}$ 99-100, 239-246.

FERRAN, A. y VALLEJO, R. (1998): «Long-term plant regeneration after wildfires in Mediterranean ecosystems of NE Spain», en Fire management and landscape ecology (Trabaud, L. V. coord.). Washington, Edit. Fairfield, 155-166.

FORBES, M. S., RAISON, R. J. y SKJEMSTAD, J. O. (2006): «Formation, transformation and transport of black carbon (charcoal) in terrestrial and aquatic ecosystems». Science of the Total Environment, $\mathrm{n}^{\circ} 370,190-206$.

GENERALITAT VALENCIANA, CONSELLERIA DE MEDI AMBIENT, AIGUA, URBANISME I HABITATGE (2009): «Estadísticas de Incendios». Disponible en http://www. cma.gva.es

GIMENO-GARCÍA, E., ANDREU, V. y RUBIO, J. L. (2000): «Changes in organic matter, nitrogen, phosphorous and cations in soil as a result of fire and fire and water erosion in a Mediterranean landscape». European Journal of Soil Science, ${ }^{\circ}$ 51, 201-210.

GIMENO-GARCÍA, E., ANDREU, V. y RUBIO, J. L. (2007): «Influence of vegetation recovery on water erosion at short and medium-term after experimental fires in a Mediterranean shrubland». Catena, ${ }^{\circ}$ 69, 150-160.

GIOVANNINI, G. y LUCCHESI, S. (1983): «Effect of fire on hydrophobic and cementing substances of soil aggregates». Soil Science, $\mathrm{n}^{\circ} 136,231-236$.

GIOVANNINI, G., LUCCHESI, S. y GIACHETTI, M. (1990a): «Effects of heating on some chemical parameters related to soil fertility and plant growth». Soil Science, $\mathrm{n}^{\circ} 149,344-$ 350 .

GIOVANNINI, G., LUCCHESI, S. y GIACHETTI, M. (1990b): «Beneficial and detrimental effects of heating on soil quality», en Fire in ecosystem dynamics: Mediterranean and northern perspectives (Goldammer, J. G. y Jenkins, M. J. coord.). The Hague, Edit. SPB Academic Publishing, 95-102

GONZÁLEZ-VILA, F. J., ALMENDROS, G., GONZÁLEZ-PÉREZ, A., KNICKER, H., GONZÁLEZ-VÁZQUEZ, R., HERNÁNDEZ, Z., PIEDRA-BUENA, A., DE LA ROSA, J. M. (2009): «Transformaciones de la materia orgánica del suelo por incendios naturales y calentamientos controlados en condiciones de laboratorio». En Efectos de los incendios forestales sobre los suelos en España. El estado de la cuestión visto por los científicos españoles (Cerdà, A. y Mataix-Solera, J. coord.). Valencia, Cátedra de divulgación de la ciencia, Universitat de València, 219-267.

INBAR, M., TAMIR, M. y WITTENBERG, L. (1998): «Runoff and erosion processes after a forest fire in Mount Carmel, a Mediterranean area». Geomorphology, n 24, 17-33.

KNOEPP, J.D., DEBANO, L.F. y NEARY, D.G. (2005): «Soil Chemistry», en Wildland fire in ecosystem. Effects of fire on soil and water (Neary D. G., Ryan K. C. y DeBano L. F., coord.). Ogden, UT. General Technical Report RMRS_GTR-42-vol. 4, United States Department of Agriculture, Forest Service and Rocky Mountain Research Station, 53-71. 
KUTIEL, P. (2006): «Fire and ecosystem heterogeneity: A Mediterranean case f study» Earth Surface Processes and Landforms, $\mathrm{n}^{\circ}$ 19, 187, 194.

KUTIEL, P. y NAVEH, Z. (1987a): «Soil properties beneath Pinus halepensis and Quercus calliprinos trees on burned and unburned mixed forest on Mt. Carmel, Israel». Forest Ecology and Management, $\mathrm{n}^{\circ} 20,11-24$.

KUTIEL, P. y NAVEH, Z. (1987b): «The effect of fire on nutrients in a pine forest soil». Plant and Soil, $\mathrm{n}^{\circ}$ 104, 269-274.

KUTIEL, P. y NAVEH, Z. (1990): «The effect of wildfire on soil nutrients and vegetation in an Aleppo pine forest on Mount Carmel, Israel», en Fire in ecosystem dynamics: Mediterranean and northern perspectives (Goldammer, J. G. y Jenkins, M. J. coord.). The Hague, Edit. SPB Academic Publishing, 85-94.

LAVEE, H., KUTIEL, P., SEGEV, M. y BENYAMINI, Y. (1995): «Effect of surface roughness on runoff and erosion in a Mediterranean ecosystem: the role of fire». Geomorpho$\log y, \mathrm{n}^{\circ} 11,227-234$.

LLORET, F., PAUSAS, J. G. y VILA, M. (2003): «Response of Mediterranean plant species to different fire regimes in Garraf Natural Park (Catalonia, Spain): field observations and modelling predictions». Plant ecology, $\mathrm{n}^{\circ}$ 167, 223-235.

LLORET, F. y ZEDLER, P. H. (2009): «The effect of forest fire on vegetation», en Fire effects on Soils and Restoration Strategies (Cerdà, A. y Robichaud, P. R., coord.). Enfield, Edit. Science Publishers, 257-295.

MACDONALD, L. H., LARSEN, I. J., BROWN, E., ROUGH, D., WELSH, M. J., PIETRASZEK, J. H., LIBOHOVA, Z. y SCHAFFRATH, K. (2008): «What causes runoff and sediment yields to increase after Wildfires?» Final Cost 634 International Conference «On- and Off-site Impacts of Runoff and Erosion» Aveiro (Portugal), 30th June - 4th July.

MARQUÉS, M. A. y MORA, E. (1992): «The influence of aspect on runoff and soil loss in a Mediterranean burnt forest (Spain)». Catena, $\mathrm{n}^{\circ}$ 19, 333-344.

MATAIX-SOLERA, J. y DOERR, S. H. (2004): «Hydrophobicity and aggregate stability in calcareous topsoils from fire-affected pine forests in southeastern Spain». Geoderma, ${ }^{\circ}$ $118,77-88$.

MATAIX-SOLERA, J. y GUERRERO, C. (2007): «Efectos de los incendios forestales sobre las propiedades edáficas», en Incendios forestales, suelos y erosión hídrica (MataixSolera, J. coord.). Alcoi, Edit. Caja Mediterráneo CEMACAM. 5-40.

MATAIX-SOLERA, J., GÓMEZ, I., NAVARRO-PEDREÑO, J., GUERRERO, C. Y MORAL, R. (2002): «Soil organic matter and aggregates affected by wildfire in a Pinus halepensis forest in Mediterranean environment». International Journal of Wildland Fire, ${ }^{\circ}$ 11: 107-114.

MATAIX-SOLERA, J., ARCENEGUI, V., GUERRERO, C., MAYORAL, A. M., MORALES, J., GONZÁLEZ, J., GARCÍA-ORENES, F. y GÓMEZ, I. (2007): «Water repellency under different plant species in a calcareous forest soil in a semiarid Mediterranean environment». Hydrological Processes, ${ }^{\circ}$ 21, 2300-2309.

MATAIX-SOLERA, J., ARCENEGUI, V., GUERRERO, C., JORDAN, M. M., DLAPA, P., TESSLER, N. y WITTENBERG, L. (2008): «Can terra rossa become water repellent by burning? A laboratory approach». Geoderma, n 147, 178-184. 
MAY, T. (1990): «Vegetation development and surface runoff after a fire in a catchment of southern Spain», en Fire in ecosystem dynamics: Mediterranean and northern perspectives, (Goldammer, J. G. y Jenkins, M. J. coord.). The Hague, Edit. SPB Academic Publishing, 117-126.

MAYOR, A. G. (2007): «Post-fire hydrological and erosional responses of a Mediterranean landscape: Seven years of catchment-scale dynamics». Catena, $\mathrm{n}^{\circ} 71,68-75$.

MAYOR, A. G., BAUTISTA, S., LLOVET, J. y BELLOT, J. (2007): «Post-fire hydrological and erosional responses of a Mediterranean landscape: Seven years of catchment-scale dynamics». Catena, $\mathrm{n}^{\mathrm{o}} 71,68-75$.

MINISTERIO DE MEDIO AMBIENTE. SUBDIRECCIÓN GENERAL DE POLÍTICA FORESTAL Y DESERTIFICACIÓN Y SECRETARÍA GENERAL PARA EL TERRITORIO Y LA BIODIVERSIDAD (2006): Los incendios forestales en España. Decenio 1996-2005.

MOLINAS, M. L. y VERDAGUER, D. (1993): «Lignotuber ontogeny in the cork-oak (Quercus suber; Fagaceae). I. Late embryo.» American journal of Botany, $\mathrm{n}^{\circ}$ 80, $172-$ 181.

MOLINERO, F., CASCOS, C., GARCÍA, A. y BARAJA, E. (2008): «Dinámica de los incendios forestales en Castilla y León». Boletín de la A. G. E., n 48, 39-70.

MONTIEL MOLINA, C. (1994): «Decadencia y degradación de las masas forestales valencianas». Investigaciones geográficas, $\mathrm{n}^{\circ} 12,185-199$

MOODY, J. A. y MARTIN, D. A. (2009): «Forest fire effects on geomorphic processes», en Fire effects on soils and restoration strategies (Cerdà, A. y Robichaud, P. R., coord.). Enfield, Edit. Science Publishers, 41-79.

MORENO, J. M., CRUZ, A., FERNÁNDEZ, F., LUNA, B., PÉREZ, B., QUINTANA, J. R. y ZUAZUA, E. (2004): «Ecología del monte mediterráneo en relación con el fuego: el jaral-brezal de Quintos de Mora (Toledo)», en Avances en el estudio de la gestión del monte mediterráneo, (Vallejo, R. y Alloza, J. A. coord.). Valencia, Edit. Fundación CEAM, 17-45.

NAVEH, Z. (1974): «Effects of fire in the Mediterranean Region», en Fire and ecosystems (Kozlowski, T. T. y Ahlgren coord.). New York, Edit. Academic Press, 139-194.

NAVEH, Z. (1975): «The evolutionary significance of fire in the Mediterranean Region». Vegetation, $\mathrm{n}^{\circ} 29,199-208$.

NAVEH, Z. (1990): «Fire in the Mediterranean - A landscape ecological perspective», en Fire in ecosystem dynamics: Mediterranean and northern perspectives (Goldammer, J. G. y Jenkins, M. J. coord.). The Hague, Edit. SPB Academic Publishing, 1-20.

NAVEH, Z. (1991): «The role of fire in Mediterranean vegetation». Botanika Chronika, $\mathrm{n}^{\circ}$ 10, 386-405.

NEARY, D. G., KLOPATEK, C., C., DEBANO, L. F. y FFOLLIOTT, P. F. (1999): «Fire effects on belowground sustainability: a review and synthesis». Forest Ecology and Management, $\mathrm{n}^{\mathrm{o}}$ 122, 51-71.

PASTOR-LÓPEZ, A. y MARTIN-MARTIN, J. (1995): Potential nitrogen losses due to fire from Pinus halepensis stands in the Alicante Province (Southeastern Spain): Mineralomass variability, in The Biswell Symposium: Fire issues and solutions in urban interface and wildland ecosystems, USDA Forest Service. 
PAUlA, S., CERVELlO, C. P. y PAUSAS, J. G. (2006): «Fire as a germination cue: A review for the Mediterranean basin». Forest Ecology and Management, $\mathrm{n}^{\circ} 234 \mathrm{~S}, \mathrm{~S} 176$.

PAUSAS, J. G., CARBO, E., NEUS-CATURLA, R., GIL, J. M. y VALLEJO, R. (1999): «Post-fire regeneration patterns in the eastern Iberian Peninsula». Acta Oecologica, $\mathrm{n}^{\circ}$ 20, 499-508.

PAUSAS, J. G. (2004a): «La recurrencia de incendios en el monte mediterráneo», en Avances en el estudio de la gestión del monte mediterráneo (Vallejo, R. coord.). Valencia, Edit. CEAM, 47-64.

PAUSAS, J. G. (2004b): «Changes in fire and climate in the eastern Iberian Peninsula (Mediterranean basin)». Climatic change, $\mathrm{n}^{\circ} 63,337-350$.

PAUSAS, J. G., LLOVET, J., RODRIGO, A. y VALLEJO, R. (2008): «Are wildfires a disaster in the Mediterranean basin?-A review». International Journal of Wildland Fire, $\mathrm{n}^{\circ} 17$, 713-723.

PAUSAS, J. G. y KEELEY, J. E. (2009): «A burning story: the role of fire in the history of life». BioScience, nº59, 593-601.

PYNE, S. (2001): Fire, a brief history.British museum press, London.

PRODON, R., FONS, R. y ATHIAS-BINCHE, F. (1987): «The impact of fire on animal communities in the Mediterranean area», en The role of fire in ecological systems (Trabaud, L. V. coord.). Den Haag, Edit. SPB Academic, 121-157.

RAISON, R. J., KHANNA, P. K. y WOODS, P. V. (1984): «Mechanisms of element transfer to the atmosphere during vegetation fires». Canadian Journal of Forest Research, $\mathrm{n}^{\circ} 15$, 132-140.

RAISON, R. J., KHANNA, P. K., JACOBSEN, K., L. S., ROMANYA, J. y SERRASOLSES, I. (2009): «Effect of fire on forest nutrient cycles», en Fire effects on soils and restoration strategies (Cerdà, A. y Robichaud, P. R., coord.). Enfield, Edit. Science Publishers, 225-256.

REYES, O. y CASAL, M. (2006): «Can smoke affect the germination of Pinus sylvestris, P. nigra, P. uncinata and P. pinaster?» Forest Ecology and Management, $\mathrm{n}^{\circ} 234 \mathrm{~S}, \mathrm{~S} 184$.

ROBICHAUD, P. R. (2009): «Post-fire stabilization and rehabilitation», en Fire effects on Soils and Restoration Strategies (Cerdà, A. y Robichaud, P. R., coord.). Enfield, Edit. Science Publishers, 299-320

ROMERO GONZÁLEZ, J. (1978): «Incendios forestales». Cuadernos de Geografía, n 23, 132-133.

SALA, M., SABATÉ, S. y GRACIA, C.A. (1990): «Organización vertical en un matorral de Quercus coccifera y Pistacia lenticus tras el fuego: índice foliar y gradientes morfológicos». Mediterránea Serie de Estudios Biológicos, $\mathrm{n}^{\circ}$ 12, 47-58.

SCOTT, A. C. (2000): «The Pre-Quaternary history of fire». Palaeo, nº 164, 281-329.

SCOTT, A. C. (2009): «Forest fire in the fossil record», en Fire effects on soils and restoration strategies (Cerdà, A. y Robichaud, P. R., coord.). Enfield, Edit. Science Publishers, 1-37.

SHAKESBY, R. A. y DOERR, S. H. (2006): «Wildfire as hydrological and geomorphological agent». Earth-Science Reviews, n $74,269-307$.

TESSLER, N., WITTENBERG, L., MALKINSON, D. y GREENBAUM, N. (2008): «Fire effects and short-term changes in soil water repellency - Mt Carmel, Israel». Catena, $\mathrm{n}^{\circ}$ 74, 185-191. 
TRABAUD, L. V. (1973): Experimental study on the effects of prescribed burning on a Quercus coccifera L. Garrigue: early results. Procediments Annual Tall Timbers Fire Ecology Conference, Tallahassee, Florida, March 22-23.

TRABAUD, L. V. (1990): «Fire resistance of Quercus coccifera L. garrigue», en Fire in ecosystem dynamics: Mediterranean and northern perspectives (Goldammer, J. G. y Jenkins, M. J. coord.). The Hague, Edit. SPB Academic Publishing, 21-32.

ÚBEDA, X., BERNIA, S. y SIMELTON, E. (2005): «The long-term effects on soil properties from a forest fire of varying intensity in a Mediterranean environment», en Catchment Dynamics and river processes: Mediterranean and other climate regions (García, C. y Batalla, R. J. coord.). Amsterdam, Edit. Elsevier 87-102.

VALLEJO, R., SERRASOLSES, J. A., ALLOZA, J. A., BAEZA, M. J., BLADÉ, C., CHIRINO, E., DUGUY, B., FUENTES, D., PAUSAS, J. G., VALDECANTOS, A., VILAGROSA, A. (2009): «Long-term restoration strategies and techniques», en Fire effects on Soils and Restoration Strategies (Cerdà, A. y Robichaud, P. R., coord.). Enfield, Edit. Science Publishers, 373-398.

VÉLEZ MUÑOZ, R. (1999): «El período 1848-1947 en la defensa contra incendios forestales en España». En Incendios históricos: una aproximación multidisciplinar (Araque Jiménez, E., coord.). Baeza, Universidad Internacional de Andalucía, 13-33. 
